\title{
The AO and MCAO for the 4m European Solar Telescope
}

\author{
Thomas Berkefeld ${ }^{a}$ and the EST AO group \\ ${ }^{a}$ Kiepenheuer-Institut für Sonnenphysik, Freiburg, Germany
}

\begin{abstract}
We give an overview of the Adaptive Optics (AO) and Multi-conjugate Adaptive Optics (MCAO) system of the planned $4 \mathrm{~m}$ European Solar Telescope (EST). The two possible configurations of the wavefront sensors are explained. The effect of pupil segmentation due to the telescope spiders is discussed.
\end{abstract}

Keywords: EST, VTT, solar adaptive optics, AO, multi-conjugate adaptive optics, MCAO, extended source wavefront sensing, pupil segmentation

\section{INTRODUCTION}

For more than 15 years adaptive optics improves observations of the sun at large solar telescopes and has become a mature tool for improving the image quality. Since solar astronomy is done to a large extent at visible wavelengths, the small corrected field of view (FoV) of only a few arcseconds limits the scientific use. In order to observe sunspots extending over more than one arc minute on sky with long slit spectrographs at diffraction-limited spatial resolution and spectral resolutions exceeding 200000 (leading to long exposures), a larger corrected FoV is required. Furthermore the strong variation of the PSF across the FoV complicates image reconstruction techniques of short exposures such as speckle imaging or multi object multi frame blind deconvolution (MOMFBD).

By adding deformable mirrors (DMs) in the conjugate heights of the atmospheric turbulence, a 3D-correction can be achieved (multi-conjugate AO), leading to much wider corrected FoV. In 2016, the first solar MCAO using three DMs successfully corrected a field of view of 30 arcseconds $^{7}$ at the $1.6 \mathrm{~m}$ telescope of the Big Bear Solar Observatory, acting as a testbed for a future DKIST MCAO. The first science observations followed in 2017. It should be noted that the MCAO mode was working more stable than the conventional AO (CAO) mode for reasons explained in section 4 .

In order to minimize the number of optical surfaces, the optical design of the planned 4m European Solar Telescope ${ }^{1}$ incorporates an MCAO system as part of the main telescope optics, leading to a very efficient design of only 14 mirrors, including five DMs and a separate tip-tilt mirror (TT).

The EST AO group consists of people from institutes in Tenerife and Oviedo (Spain), Freiburg (Germany), Durham (UK), Stockholm (Sweden), Locarno and Yverdon-les-Bains (Switzerland) and Rome (Italy). It has formed several years ago in order to address open questions such as the best wavefront sensor (WFS) concept, order of the DMs, reconstruction issues etc. with increasing level of detail. Numerical simulations now propagate (artificial) solar images through the atmosphere and DM models, allowing very accurate predictions of the system performance.

Table 1 shows the basic parameters and science requirements for the $\mathrm{EST}^{1}$ concerning the image quality after (MC)AO correction. Certain parameters such as the actuator pitch of the layer DMs are not fixed yet, shown are the baseline values used in the simulations. The most challenging specs are the $30 \%$ Strehl ratio at $r_{0}=7 \mathrm{~cm}$ and $60 \%$ Strehl at $r_{0}=20 \mathrm{~cm}$ across a corrected MCAO FoV of one arcminute for a typical turbulence height profile. Due to the visible wavelength regime and the typical high zenith angle observations, these requirements pose a real challenge.

A summary of the EST MCAO system performance simulations and an analysis of the proper order of the DMs can be found in papers by Montilla ${ }^{5}$ and Montoya. ${ }^{8}$ They show that the science requirements can be met for typical elevations. 


\begin{tabular}{|l|l|}
\hline telescope & $4 \mathrm{~m}$ on-axis Gregory \\
\hline \#DMs + heights (current design) & TT +5 DMs @ $0,5,9,12,25 \mathrm{~km}$ \\
\hline actuator pitch projected on the pupil & $8,30,30,30,30 \mathrm{~cm}$ \\
\hline science FoV & 120 arsec square \\
\hline MCAO-corrected FoV & 60 arcsec \\
\hline MCAO performance (specs) & $\mathrm{S}=0.3$ for $r_{0}=7 \mathrm{~cm}$, avg. $C_{n}^{2}$ profile \\
& $\mathrm{S}=0.6$ for $r_{0}=20 \mathrm{~cm}$, avg. $C_{n}^{2}$ profile \\
\hline
\end{tabular}

Table 1. Parameters of the EST MCAO

\section{THE PARAMETER SPACE OF A SOLAR MCAO SYSTEM}

The main parameters of a solar MCAO system are - as in night-time MCAO - number and conjugate height of the DMs plus their actuator pitch. Furthermore, two wavefront sensor concepts exist, both using a Shack-Hartmann correlating WFS.

\subsection{Number, heights and order of DMs}

Similar to night-time MCAO, the number of layer DMs and actuators per DM is a function of the specified image quality at the science focus for a given turbulence profile. However, solar MCAO faces the challenge of small $\mathrm{r}_{0}$ s due to visible science wavelenghts and observations at high air masses due to observartions early in the morning, requiring more DMs at higher conjugate heights. The smaller actuator pitch in combination with the large DM heights leads to more dynamic misregistration between DMs and WFSs.

Fig. 1 shows the optical scheme of the 4m EST: M1 and M2 are part of the Gregory-type main optics, with F1 housing a field stop that reflects ca. $99 \%$ of the light (2 arcmin square FoV). M5 and M8 are the collimator and the reimager of the conventional $\mathrm{AO}$ which consists of the tiptilt mirror $\mathrm{M} 6$ and the ground layer $(\mathrm{AO})$ DM M7. In order to allow sufficiently large layer (MCAO) DMs, M8 changes the focal ratio to f/40. The four MCAO DMs M9-M12 are at conjugate heights of (presently) 25,12,9 and $5 \mathrm{~km}$. M13 generates the final f/50 beam. It can be seen that the MCAO layer $\mathrm{DMs}$ are not in reverse order (which would require reimaging for each DM) but are in the natural order after a focus (high to low altitiude). Since the ground layer turbulence is strongest, pupil DM and TT-mirror come first. This concept has been validated by Montoya. ${ }^{8}$ The night-time WFS position is shown in blue (3x3 CAO sensors), the VTT WFS positions are shown in black (one CAO sensor and one wide-field sensor).

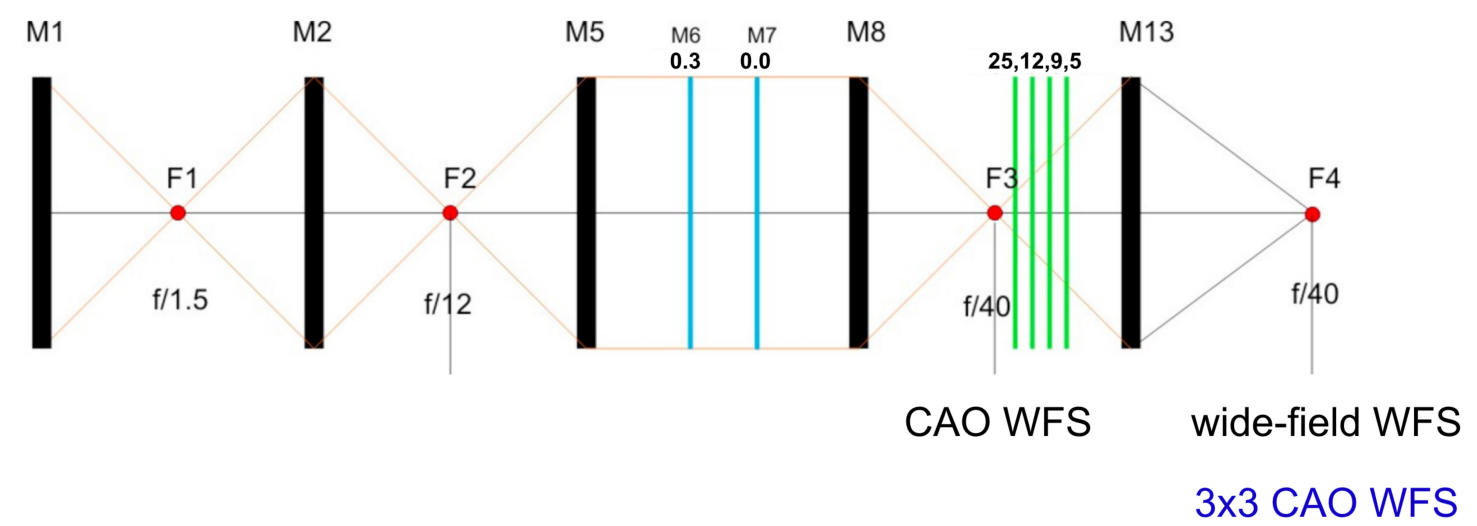

Figure 1. EST Optical scheme

\subsection{The Wavefront Sensor}

Solar WFSs use crosscorrelating Shack-Hartmann sensors working on images of the solar surface, typically crosscorrelating subaperture images against a reference subaperture image across a FoV of 10-12 arcseconds. The crosscorrelation methods have been compared by Löfdahl. ${ }^{2}$ 


\subsubsection{The Use of Low-Contrast Granulation as a Target}

In order to estimate the RMS signal (image contrast in the WFS), the power spectrum of the solar granulation has to be multiplied by the MTF of a WFS subaperture and the MTF of the turbulent atmosphere within the spatial regime that WFS can sense. This reduces the original solar contrast of $13-14 \%$ at $525 \mathrm{~nm}$ to a few percent, depending on the seeing and the subaperture size. The RMS noise is basically the photon noise: Assuming a full well capacity of 60ke- (50ke- used), the photon noise is 220 e- RMS. This is much more than the digitization noise of an 8-bit camera (ca 250e- peak-to-peak, ca 80e- RMS) and the read-out noise. Therefore, for solar AO systems, 8-bit WFS cameras are sufficient (if the light level is kept close to the full well capacity).

In order to get a high contrast onto the WFS camera, it is absolutely mandatory to have smooth telescope optics, i.e. no mid-frequency ripples on optical surfaces that cannot be resolved by the WFS but reduce the contrast. Furthermore the WFS camera should have a high full well capacity. Although in numerical simulations even cameras with full well capacities as low as 25000e- work well, operation of existing AO systems shows that a higher full well capacity is advantageous. Experience with existing AO systems shows that a well-working AO requires a contrast of at least 3\%. Examples of well-working and not so well-working images and correlation functions are shown in Fig. 2.
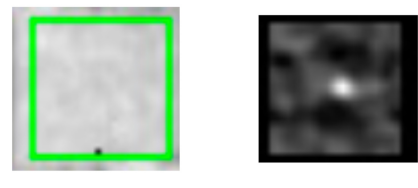

high contrast (> 3\%): decent seeing AND good optics
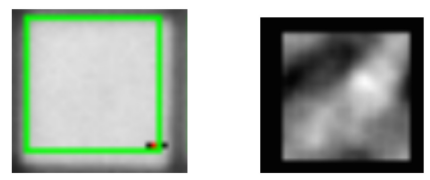

\section{low contrast $(<2 \%)$ : bad seeing OR bad optics}

Figure 2. . Good (top) and bad (bottom) images (left) and resulting correlation functions (right) of solar granulation through a single lenslet

As can be seen in fig. 3, decreasing the subaperture size below $8 \mathrm{~cm}$ does not result in much gain in performance any more. The science requirements of $30 \%$ Strehl ratio at $r_{0}=7 \mathrm{~cm}, 60 \%$ Strehl at $r_{0}=20 \mathrm{~cm}$ can easily be met for the center of the corrected FoV (CAO mode). The optimal pupil sampling (subaperture size / actuator

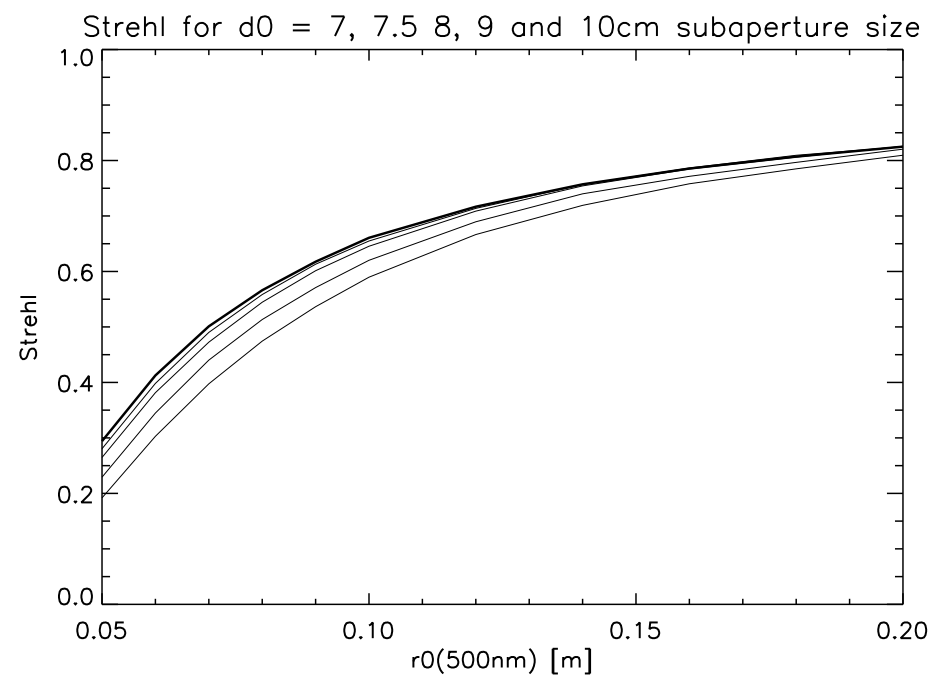

Figure 3. Strehl as a function of $r_{0}$ and subaperture size(=actuator spacing) of $d=7,7.5,8,9,10 \mathrm{~cm}$ (top to bottom) 
pitch for Fried geometry) for a conventional solar AO is determined by the tradeoff of increasing measurement noise (due to the decreasing contrast transfer of smaller subapertures) and decreasing fitting error.

\subsubsection{Extended WFS FoV}

Since the correlating SH-WFS works on granulation, it must have an extended FoV. For conventional solar $\mathrm{AO}$, the FoV is typically of the order of 10-12 arcseconds, covering several granules and thus providing a stable correlation function. As can be seen in Fig. 4, the metapupil of a subaperture increases with height, leading to decreased sensitivity of high altitude turbulence, solar AO systems therefore always have the characteristics of a ground layer AO. Furthermore, the non-zero-FoV solar WFS "sees" more actuators of high altitude DMs than it would be the case for the stellar zero-FoV WFS. The calibration of the MCAO-DMs (measurement of the interaction matrix) has therefore to be done with an extended target in a preceding focus.

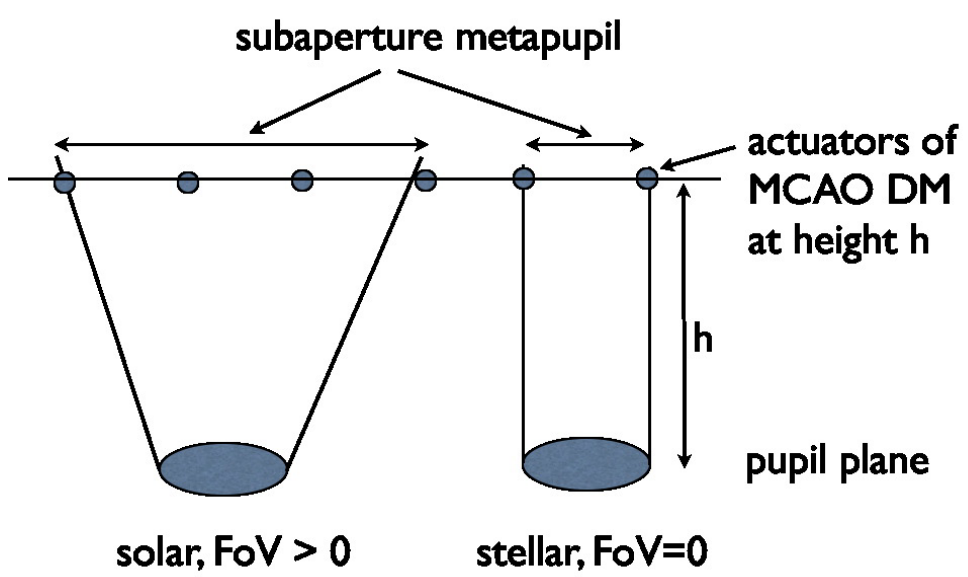

Figure 4. metapupil size of a subaperture for $\mathrm{FoV}>0$ (left) and $\mathrm{FoV}=0$ (right) at high altitudes. The metapupil for FoV $>0$ is much larger than in the FoV $=0$ case. As a consequence, a FoV $>0$ also "sees" more actuators of a high altitude MCAO-DM, leading to underestimation of high spatial frequency errors at high altitude.

\subsubsection{The two WFS concepts}

Two WFS concepts are being investigated, both using correlating Shack-Hartmann sensors:

1. night-time approach: use several (e.g. 3x3) CAO (high order, narrow FoV) sensors covering the MCAO corrected FoV. The sensors are downstream of all DMs.

Advantages:

- since the strong ground layer is sensed by every CAO sensor, averaging leads to a much improved SNR for measuring the strong ground layer as compared to a single CAO sensor. This is the reason why the MCAO at the Big Bear Solar Observatory runs more stable than the CAO.

- the influence / shape of all layer DMs is sensed directly with the high spatial sampling of the CAO sensors.

Disadvantages:

- CAO sensor downstream of the layer DMs is more susceptible to dynamic misregistration because of its smaller subaperture size.

- as many sensors as guide regions required (e.g. $3 \times 3$ ), complex optics to separate the beams into the sensors 
2. VTT concept: use one CAO (high order, narrow FoV) sensor covering the center of the FoV, use one MCAO (low order, wide FoV) for covering the full MCAO corrected FoV. The CAO sensor is upstream of the layer DMs and downstream of the pupil DM, the MCAO is downstream of all DMs. This concept is described in $^{4}$ and has been used successfully during first tests at the VTT in 2005 (with only one layer $\mathrm{DM})$.

Advantages:

- less prone to dynamic misregistration because only the wide field (MCAO) sensor featuring its large subapertures is downstream of the layer DMs.

- free asterism, any number of guide regions

- only two sensors, however, it might be advantageous to split the MCAO sensor into four (pupil quadrants) if camera technology does not provide cameras with more than two GPixel per second.

Disadvantages:

- strong ground layer turbulence sensed only by one sensor (as in the CAO case).

- position of CAO sensor requires downstream optics, especially layer DMs, to be free of aberrations of spacial frequencies not seen by the MCAO sensor.

First simulations and lab experiments (with two layer DMs) have shown that both concepts work. On sky, the night-time setup has been shown to work very well at the Big Bear Solar Observatory (the conjugate height of the uppermost DM was $8 \mathrm{~km}$, therefore no problems with dynamic misregistration). The VTT setup also worked (within its limited capabilities in 2005). The choice between the two concepts one of the most important in the whole MCAO system and must be proven by both numerical simulations and (preferably on-sky) experiment. The simulations face the problem that the dynamic misregistration must be included which is very compute intensive.

Table 2 lists the parameters of the two WFSs:

\begin{tabular}{|l|c|c|}
\hline & CAO-WFS & wide-field WFS \\
\hline FoV [arcsec] & $10-12$ & $60-70$ \\
\hline \# guide region & 1 & any, 19 work well \\
\hline crosscorrelation FoV $[$ arcsec] & $10-12$ & $10-12$ \\
\hline subaperture size $[\mathrm{cm}]$ & 8 & $20-30$ \\
\hline
\end{tabular}

Table 2. wavefront sensor parameters. The night-time approach uses several (e.g. 3x3) CAO sensors covering the MCAOcorrected FoV, the VTT approach uses one CAO at the center of the corrected FoV plus one wide field sensor covering the full MCAO-corrected FoV.

\section{THE SPIDER PROBLEM}

A huge concern for obstructed telescopes with $\mathrm{AO}$ correction are the telescope spiders. In contrast to the pyramid sensors of night-time AO systems using the full aperture, the (correlating) Shack-Hartmann sensors of solar AO systems subdivide the telescope pupil, leading to unsensed wavefront gradients under the spiders. If their width approaches the size of $r_{0}$, then the phasing errors in the wavefront reconstruction, ${ }^{6,10}$ result in overall wavefront errors of dozens of nanometers for typical (daytime / visible wavelengths) conditions. Therefore it is mandatory to keep the spider width as small as possible. At solar telescopes, keeping the prime focus cooling hoses within the shadow of the spiders poses a challenge. All EST spiders will have a maximum width of $7 \mathrm{~cm}$ (projected on the $4 \mathrm{~m}$ pupil), leading to small but measurable wavefront errors.

Another inconvenience is due to flatfielding: Since the EST spiders block certain subapertures almost completely, two different flatfields at different spider (pupil) rotation angles must be taken and the brighter of the two must be used for each subaperture. In real live this requires the sequence pupil alignment, flatfield 1, pupil rotation by $45 \mathrm{deg}$, pupil realignment, flatfield 2, pupil rotation to desired angle, pupil realignment. 
atmospheric phase

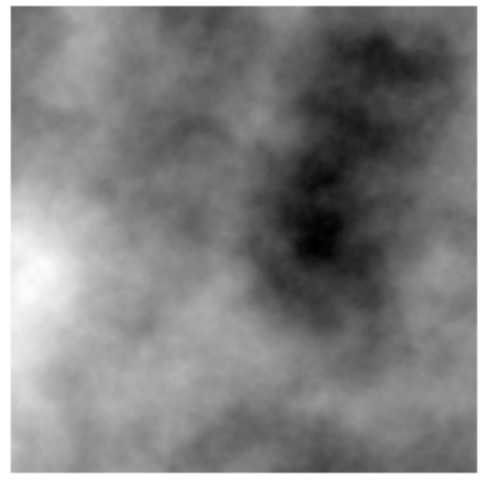

telescope pupil with spiders

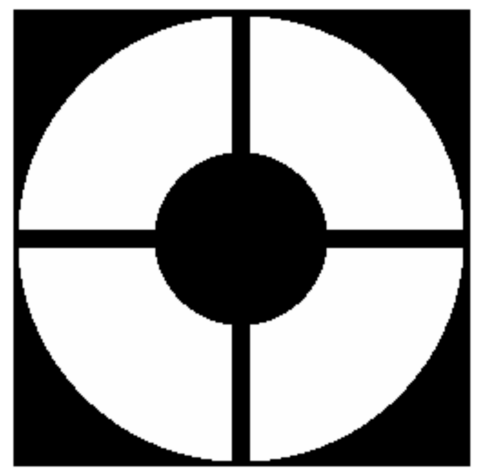

phasing error after $\mathrm{AO}$ correction

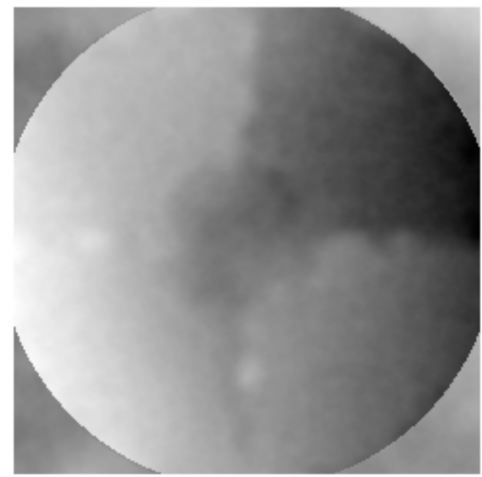

Figure 5. The problem of pupil segmentation: left: incoming aberrated wavefront, center: pupil geometry, spider width exaggerated, right: wavefront after $\mathrm{AO}$ correction. A piston difference occurs in one of the quadrants.

\section{WAVEFRONT RECONSTRUCTION}

The baseline wavefront reconstructors being investigated are the $\mathrm{FRIM}^{9}$ and $\mathrm{MMSE}^{6}$ reconstructors. Simple SVD reconstructors do not work well even for conventional AO because of the spider problem, instead, the reconstructor must be regularized with respect to the turbulence statistics. Furthermore, subapertures which cannot be used should be removed from the reconstruction completely. ${ }^{6}$ In addition, the possible use of neuronal networks is being investigated.

One obstacle for MCAO wavefront reconstruction are the different rotations inside the EST telescope: The spiders rotate with respect to all DMs, in addition, DM0 rotates with respect to the layer DMs. A possible simplification would be an adaptive secondary mirror which would eliminate the rotation between the spiders and DM0, resulting in non-rotating spiders for the Fried geometry between DM0 and the CAO WFS(s).

\section{FUTURE WORK}

The next tasks will be to

- find out which of the two wavefront sensor concepts works better, including the effects of misregistration and pupil segmentation. Simulations will be checked by future lab- and on-sky experiments.

- compare reconstructors (MMSE, FRIM, neural networks)

- fine tune certain parameters such as the actuator pitch of the layer DMs

- check whether the various rotations inside the telescope pose a problem (frequent reconstructor update).

In order to get the required manpower, i.e. additional funding, we will write a proposal for the SOLARNET II framework.

\section{REFERENCES}

1. Collados, M., "EST: The European Solar Telescope", 12th European Solar Physics Meeting, Freiburg, Germany, 2008, Online at http://espm.kis.uni-freiburg.de/fileadmin/user_upload/espm/Proceedings-Talks/t_604.pdf

2. Löfdahl, M., "Evaluation of image shift measurement algorithms for solar ShackHartmann wavefront sensors", submitted to Astronomy \& Astrophysics 2010

3. Soltau, D., Berkefeld, T., Sanchez Capuchino, J., Collados Vera, M., del Moro, D., Löfdahl, M., Scharmer, G., "Adaptive Optics and MCAO for the 4-m European Solar Telescope EST", Proc. SPIE 7736 (2010) 
4. Berkefeld, T., Soltau, D., von der Lühe, O., "Results of the multi-conjugate adaptive optics system at the German solar telescope, Tenerife", in Astronomical Adaptive Optics Systems and Applications, Proc. SPIE 5903, 219-226 (2005).

5. Montilla, I., Montoya, L., "The MCAO system of the EST: DM height determination for best performance using real daytime statistical turbulence data", these proceedings

6. Bonnefond, S., Tallon, M., Le Louarn, M., Madec, P.Y., "Wavefront reconstruction with pupil fragmentation: study of a simple case", Proc. SPIE 9909, pp. 9909-287 (2016).

7. Schmidt, D., Gorceix, N., Goode, P., Marino, J., Rimmele, T., Berkefeld, T., Wöger, F., Zhang, X., Rigaut, F., von der Lühe, O., "Clear: advancing wide-field adaptive optics for observations of the Sun", these proceedings

8. Montoya, L.,Montilla, I., Collados, M., "MCAO numerical simulations for EST: analysis and parameter optimization", AO4ELT4 (2015)

9. Bechet, C., Tallon, M., Thiebaut, E., "FRIM: minimum-variance reconstructor with a fractal iterative method", Proc. SPIE 6272 (2006).

10. Obereder, A., "On the performance of reconstruction methods in the presence of spiders", these proceedings 